\title{
THE FAT LOCUS OF HILBERT SCHEMES OF POINTS
}

\author{
MARC COPPENS
}

(Communicated by Louis J. Ratliff, Jr.)

\begin{abstract}
Let $X$ be a smooth projective variety over an algebraically closed field $k$. Let Hilb $^{d}(X)$ be its Hilbert scheme of 0-dimensional subschemes of $X$ of degree $d$. Let [Hilb $\left.{ }^{d}(X)\right](k)$ be the set of $k$-rational points. We prove that the subset of points of $\left[\mathrm{Hilb}^{d}(X)\right](k)$ corresponding to fat points on $X$ is a locally closed subset with respect to the Zariski topology.
\end{abstract}

Let $X$ be a smooth $d$-dimensional projective variety defined over an algebraically closed field $k$. For $n \in \mathbb{Z}_{\geq 1}$ let $\operatorname{Hilb}^{n}(X)$ be the Hilbert scheme parametrizing 0-dimensional subschemes $Z$ of $X$ of degree $\operatorname{deg}(Z)=n$ (i.e., $\sum\left(l\left(\mathscr{O}_{Z ; P}\right): P \in X(k)\right)=n$, where $X(k)$ is the set of the closed points on $X$ and $l$ means length). We also write $Z$ for the corresponding point on $\left[\operatorname{Hilb}^{n}(X)\right](k)$.

Definition. A 0-dimensional closed subscheme $Z$ of $X$ is called fat of type $\left(n_{1} ; n_{2} ; \ldots ; n_{t}\right)$ if $n_{1} \geqslant n_{2} \geqslant \cdots \geqslant n_{t} \geqslant 1$ and there exist $t$ different points $P_{1} ; \ldots ; P_{t}$ on $X(k)$ such that

$$
\begin{aligned}
& \mathscr{I}_{Z ; P}=\mathscr{O}_{X ; P} \quad \text { for } P \in X(k) \backslash\left\{P_{1} ; \ldots ; P_{t}\right\}, \\
& \mathscr{I}_{Z ; P_{i}}=\left(\mathscr{M}_{X ; P_{i}}\right)^{n_{i}}
\end{aligned}
$$

where $\mathscr{I}_{Z}$ is the sheaf of ideals of $Z \subset X$ and $\mathscr{M}_{X ; P_{i}}$ is the maximal ideal in the local ring $\mathscr{O}_{X ; P_{i}}$ of $X$ at $P_{i}$.

Since $X$ is smooth, we have $\operatorname{deg}(Z)=\sum\left(\left({ }^{n_{i}+d-1} d\right): 1 \leqslant i \leqslant t\right)$ in the above definition. For $n=\sum\left(\left(\begin{array}{c}n_{i}+d-1 \\ d\end{array}\right): 1 \leqslant i \leqslant t\right)$ we define

$$
\operatorname{Hilb}\left(n_{1} ; \ldots ; n_{t}\right)=\left\{Z \in\left(\operatorname{Hilb}^{n}(X)\right)(k): Z \text { is fat of type }\left(n_{1} ; \ldots ; n_{t}\right)\right\} \text {. }
$$

We call $\operatorname{Hilb}\left(n_{1} ; \ldots ; n_{t}\right)$ the fat locus of type $\left(n_{1} ; \ldots ; n_{t}\right)$. In this paper, we prove

Theorem. $\operatorname{Hilb}\left(n_{1} ; \ldots ; n_{t}\right)$ is a locally closed subset of $\left[\operatorname{Hilb}^{n}(X)\right](k)$, i.e., it is the intersection $Z \cap U$ of a closed subset $Z$ and an open subset $U$ of $\left[\operatorname{Hilb}^{n}(X)\right](k)$. (Of course, we work with respect to the Zariski topology.)

Received by the editors November 25, 1991.

1991 Mathematics Subject Classification. Primary 14C05.

The author is affiliated with the University at Leuven (Celestijnenlaan 2000, B3030 Leuven, Belgium) as a research fellow. 
The motivation comes from a growing interest in fat points (see, e.g., [1, 2]). In particular, it is proved in [5] that $\operatorname{Hilb}\left(n_{1} ; n_{2} ; \ldots ; n_{t}\right)$ is an irreducible constructible subset of $\left[\operatorname{Hilb}^{n}(X)\right](k)$ (it is only stated for the case $X=\mathbb{P}^{2}$ but his proof works in our generality), and the theorem proved in this paper is also mentioned in [5] as a well-known but not yet proved statement.

Proof. For $m \in \mathbb{Z}_{\geq 1}$ and $n=\left(\begin{array}{c}m+d-1 \\ d\end{array}\right)$ it is remarked in [5] that $\mathrm{Hilb}(m)$ is a closed subset of $\left[\mathrm{Hilb}^{n}(X)\right](k)$. So we can assume $t \geqslant 2$ and are going to use induction on $t$. We abbreviate $\left(n_{1} ; \ldots ; n_{t}\right)$ by $\mathbf{n}$ and $\operatorname{Hilb}\left(n_{1} ; \ldots ; n_{t}\right)$ by $\operatorname{Hilb}(\mathbf{n})$.

Lemma 1. For each irreducible component $H$ of $\left[\mathrm{Hilb}^{n}(X)\right](k)$ it is enough to prove that $H \cap \mathrm{Hilb}(\mathbf{n})$ is locally closed in $H$.

Proof. Let $H^{1}, \ldots, H^{s}$ be the irreducible components of $\operatorname{Hilb}^{n}(X)$ and let $H^{i}(\mathbf{n})=H^{i} \cap \operatorname{Hilb}(\mathbf{n})$. Since $H^{i}(\mathbf{n})$ is locally closed in $H^{i}$, we have $H^{i}(\mathbf{n})=$ $U_{i}^{\prime} \cap C_{i}$, where $U_{i}^{\prime} \subset H^{i}$ is open and $C_{i} \subset H^{i}$ is closed. Since $H^{i}$ is closed in $\left[\operatorname{Hilb}^{n}(X)\right](k)$, one has that $C_{i} \subset\left[\operatorname{Hilb}^{n}(X)\right](k)$ is closed. Moreover, there exists $U_{i} \subset\left[\mathrm{Hilb}^{n}(X)\right](k)$ open with $U_{i}^{\prime}=U_{i} \cap H^{i}$. We can assume $U_{i} \supset$ $\left[\operatorname{Hilb}^{n}(X)\right](k) \backslash H^{i}$.

Let $U=U_{1} \cap \cdots \cap U_{s} \subset\left[\operatorname{Hilb}^{n}(X)\right](k)$ be open and $C=C_{1} \cup \cdots \cup C_{s} \subset$ [Hilb $\left.{ }^{n}(X)\right](k)$ be closed. Take $Z \in \operatorname{Hilb}(\mathbf{n})$ and let $1 \leqslant i \leqslant s$. If $Z \notin H^{i}$ then $Z \in U_{i}$. If $Z \in H^{i}$ then $Z \in H^{i}(\mathbf{n}) \subset U_{i}^{\prime} \cap C_{i}$, so $Z \in U_{i}$ and $Z \in C_{i}$. This already proves $Z \in U_{1} \cap \cdots \cap U_{s}=U$ and $Z \in C_{1} \cup \cdots \cup C_{s}=C$, and we prove $\operatorname{Hilb}(\mathbf{n}) \subset U \cap C$.

Now, let $Z \in U \cap C$. Since $Z \in C=C_{1} \cup \cdots \cup C_{s}$, there exists $1 \leqslant i \leqslant l$ with $Z \in C_{i} \subset H^{i}$. Since $Z \in U=U_{1} \cap \cdots \cap U_{s}$, we also have $Z \in U_{i}$, so $Z \in U_{i} \cap H^{i}=U_{i}^{\prime}$. This proves $Z \in U_{i}^{\prime} \cap C_{i}=H^{i}(\mathbf{n})$, hence $Z \in \operatorname{Hilb}(\mathbf{n})$. This completes the proof that $\operatorname{Hilb}(\mathbf{n})=U \cap C$.

Now we take an irreducible component $H$ of $\operatorname{Hilb}^{n}(X)$, and we let $H(\mathbf{n})=$ $H \cap \mathrm{Hilb}(\mathbf{n})$. We are going to prove that $H(\mathbf{n})$ is locally closed in $H(k)$. The restriction to $H$ of the universal family of closed subschemes of $X$ over $\operatorname{Hilb}^{n}(X)$ gives us

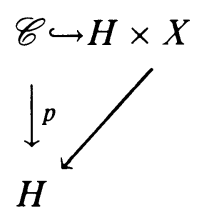

Let $\mathscr{I}_{\mathscr{C}}$ be the sheaf of ideals of $\mathscr{C}$ in $\mathscr{O}_{H \times X}$. Consider the following diagram $\left(\mathrm{pr}_{i j}\right.$ means projection on the $i$ th and $j$ th factors):

$$
\begin{aligned}
& H \times X \stackrel{\mathrm{pr}_{13}}{\longleftarrow} H \times X \times X \stackrel{\mathrm{pr}_{23}}{\longrightarrow} X \times X \\
& \downarrow \mathrm{pr}_{12} \\
& H \times X
\end{aligned}
$$

Let $\mathscr{I}_{\Delta}$ be the sheaf of ideals of the diagonal $\Delta \subset X \times X$. Let $\mathscr{I}_{n_{1} \Delta}$ be the image of $\mathscr{I}_{\Delta}^{\otimes n_{1}}$ in $\mathscr{O}_{X \times X}$. Let $\mathscr{I}_{n_{1} \Delta}^{\prime}=\mathscr{I}_{n_{1} \Delta} \cdot \mathscr{O}_{H \times X \times X}$ using $\mathrm{pr}_{23}$ and let $\mathscr{I}_{\mathscr{C}}^{\prime}=\mathscr{I}_{\mathscr{C}} \cdot \mathscr{O}_{H \times X \times X}$ using $\operatorname{pr}_{13}$ (see [3,p. 163]). Let $\mathscr{I}$ be the sum $\mathscr{I}_{n_{1} \Delta}^{\prime}+\mathscr{I}_{\mathscr{C}}^{\prime}$ 
in $\mathscr{O}_{H \times X \times X}$, and let $\mathscr{S} \subset H \times X \times X$ be the associated closed subscheme. Consider the restriction of $\mathrm{pr}_{12}$,

$$
q: \mathscr{S} \rightarrow H \times X .
$$

Lemma 2. For $(Z ; P) \in H(k) \times X$, the scheme-theoretic inverse image $q^{-1}(Z ; P)$ is the closed subscheme of $X$ with sheaf of ideals $\mathscr{I}(Z ; P)$ given by

$$
\begin{aligned}
& \mathscr{I}(Z ; P)_{Q}=\mathscr{O}_{X ; Q} \quad \text { for } Q \in X(k) \backslash\{P\}, \\
& \mathscr{I}(Z ; P)_{P}=\mathscr{I}_{Z ; P}+\mathfrak{M}_{X ; P}^{n_{1}} .
\end{aligned}
$$

Proof. For $Q \in X(k) \backslash\{P\}$ one has $\left(\mathscr{I}_{n_{1} \Delta}\right)_{(P ; Q)}=\mathscr{O}_{X \times X ;(P ; Q)}$ so $\left(\mathscr{I}_{n_{1} \Delta}^{\prime}\right)_{(Z ; P ; Q)}$ $=\mathscr{O}_{H \times X \times X ;(Z ; P ; Q)}$. Thus $\mathscr{J}_{(Z ; P ; Q)}=\mathscr{O}_{H \times X \times X ;(Z ; P ; Q)}$ and, therefore, also $\mathscr{I}(Z ; P)_{Q}=\mathscr{O}_{X ; Q}$. On the other hand, let $t_{1}, \ldots, t_{d}$ be a system of local parameters in $\mathscr{O}_{X ; P}$. This induces a system of parameters $t_{1}^{(1)}, \ldots, t_{d}^{(1)}, t_{1}^{(2)}$, $\ldots, t_{d}^{(2)}$ in $\mathscr{O}_{X \times X ;(P ; P)}$ and $\left(\mathscr{I}_{n_{1} \Delta}\right)_{(P ; P)}=\left\langle t_{1}^{(1)}-t_{1}^{(2)}, \ldots, t_{d}^{(1)}-t_{d}^{(2)}\right\rangle^{n_{1}}$, so also $\left(\mathscr{J}_{n_{1} \Delta}^{\prime}\right)_{(Z ; P ; P)}$ is generated by the images of degree $n_{1}$-monomials in $t_{i}^{(1)}-t_{i}^{(2)}$ $(1 \leqslant i \leqslant d)$ in $\mathscr{O}_{H \times X \times X ;(Z ; P ; P)}$. Moreover, $\left(\mathscr{I}_{\mathscr{C}}^{\prime}\right)_{(Z ; P ; P)}$ can be described as follows. Consider the natural local homomorphism

$$
\mathscr{O}_{H \times X ;(Z ; P)} \stackrel{\mathrm{pr}_{13}^{*}}{\longrightarrow} \mathscr{O}_{H \times X \times X ;(Z ; P ; P)} .
$$

Then $\left(\mathscr{I}_{\mathscr{C}}^{\prime}\right)_{(Z ; P ; P)}$ is induced by $\left(\mathscr{I}_{\mathscr{C}}\right)_{(Z ; P)}$. Let $\mathfrak{M}_{(Z ; P ; P)}^{\prime}$ be the ideal induced by $\mathfrak{M}_{H \times X ;(Z ; P)}<\mathscr{O}_{H \times X ;(Z ; P)}$ under

$$
\mathscr{O}_{H \times X ;(Z ; P)} \stackrel{\mathrm{pr}_{12}^{\prime \prime}}{\longrightarrow} \mathscr{O}_{H \times X \times X ;(Z ; P ; P)} \text {. }
$$

Then $(\mathscr{I}(Z ; P))_{P}=\left(\mathfrak{M}_{(Z ; P ; P)}^{\prime}+\left(\mathscr{I}_{\mathscr{C}}^{\prime}\right)_{(Z ; P ; P)}+\left(\mathscr{I}_{n_{1} \Delta}^{\prime}\right)_{(Z ; P ; P)}\right) / \mathfrak{M}_{(Z ; P ; P)}^{\prime}$ in $\mathscr{O}_{H \times X \times X ;(Z ; P ; P)} / \mathfrak{M}_{(Z ; P ; P)}^{\prime}=\mathscr{O}_{X ; P}$. Let $\mathfrak{M}^{\prime}(Z)$ be induced by $\mathfrak{M}_{H ; Z}$ un$\operatorname{der} \mathscr{O}_{H ; Z} \stackrel{\operatorname{pr}_{1}^{\prime \prime}}{\rightarrow} \mathscr{O}_{H \times X \times X ;(Z ; P ; P)}$. Then $\mathfrak{M}^{\prime}(Z)+\left(\mathscr{I}_{\mathscr{C}}^{\prime}\right)_{(Z ; P ; P)}$ is obtained from $\mathfrak{M}^{\prime \prime}(Z)+\mathscr{I}_{\mathscr{C} ;(Z ; P)}$ under $\mathscr{O}_{H \times X ;(Z ; P)} \stackrel{\operatorname{pr}_{13}^{\prime \prime}}{\rightarrow} \mathscr{O}_{H \times X \times X ;(Z ; P ; P)}$, where $\mathfrak{M}^{\prime \prime}(Z)$ is obtained from $\mathfrak{M}_{H ; Z}$ under $\mathscr{O}_{H ; Z} \stackrel{\mathrm{pr}_{1}^{\sharp}}{\rightarrow} \mathscr{O}_{H \times X ;(Z ; P)}$. Since

$$
\left(\mathfrak{M}^{\prime \prime}(Z)+\mathscr{I}_{\mathscr{C} ;(Z ; P)}\right) / \mathfrak{M}^{\prime \prime}(Z)=\mathscr{I}_{Z ; P}<\mathscr{O}_{H \times X ;(Z ; P)} / \mathfrak{M}^{\prime \prime}(Z)=\mathscr{O}_{X ; P},
$$

one has

$$
(\mathscr{I}(Z ; P))_{P}=\left(\mathscr{I}_{(Z ; P)}^{\prime}+\mathfrak{M}_{(P ; P)}^{\prime}+\left(\mathscr{I}_{n_{1} \Delta}\right)_{(P ; P)}\right) / \mathfrak{M}_{(P ; P)}^{\prime},
$$

where $\mathscr{I}_{(Z ; P)}^{\prime}$ (resp. $\left.\mathfrak{M}_{(P ; P)}^{\prime}\right)$ is induced from $\mathscr{I}_{Z ; P}$ (resp. $\mathfrak{M}_{X ; P}$ ) under $\mathscr{O}_{X ; P} \stackrel{\mathrm{pr}_{2}^{\sharp}}{\rightarrow} \mathscr{O}_{X \times X ;(P ; P)} \quad$ (resp. $\left.\mathscr{O}_{X ; P} \stackrel{\mathrm{pr}_{1}^{\sharp}}{\rightarrow} \mathscr{O}_{X \times X ;(P ; P)}\right)$. From the description of $\left(\mathscr{I}_{n_{1} \Delta}\right)_{(P ; P)}$ we obtain

$$
(\mathscr{I}(Z ; P))_{P}=\mathscr{I}_{Z ; P}+\left\langle t_{1}, \ldots, t_{d}\right\rangle^{n_{1}}=\mathscr{I}_{Z ; P}+\mathfrak{M}_{X ; P}^{n_{1}} .
$$

Remember the concept of a flattening stratification of $\mathscr{I}_{\mathscr{S}}$ over $H \times X$ (under q) (see [4, Lecture 8;6, §6]). This implies that $H \times X$ is the union of finitely many locally closed subsets where each such subset corresponds to some prescribed degree for the fibre of $\mathscr{S}$. Clearly $H^{\prime}\left(n_{1}\right)=\{(Z ; P) \in$ $\left.H \times X: \operatorname{deg}\left(\mathscr{S}_{(Z ; P)}\right)=\left(\begin{array}{c}n_{1}+d-1 \\ d\end{array}\right)\right\}$ is one such stratum (here $\mathscr{S}_{(Z ; P)}$ is the associated closed subscheme of $X \times \operatorname{Spec}(k) \operatorname{Spec}(k(Z ; P))$. 
Lemma 3. $(Z ; P) \in\left[H^{\prime}\left(n_{1}\right)\right](k)$ if and only if $\mathscr{I}_{Z ; P} \subset \mathfrak{M}_{X ; P}^{n_{1}}$.

Proof. Since $(\mathscr{I}(Z ; P))_{P} \supset \mathfrak{M}_{X ; P}^{n_{1}}$ and $l\left(\mathscr{O}_{X ; P} / \mathfrak{M}_{X ; P}^{n_{1}}\right)=\left(\begin{array}{c}n_{1}+d-1 \\ d\end{array}\right)$, one has $(Z ; P) \in H^{\prime}\left(n_{1}\right)$, i.e., $l\left(\mathscr{O}_{X ; P} /\left(\mathscr{I}(Z ; P)_{P}\right)\right)=\left(\begin{array}{c}n_{1}+d-1 \\ d\end{array}\right)$, iff $(\mathscr{I}(Z ; P))_{P}=$ $\mathfrak{M}_{X ; P}^{n_{1}}$. Because of Lemma 2 , this is equivalent to $\mathscr{I}_{Z ; P} \subset \mathfrak{M}_{X ; P}^{n_{1}}$.

Lemma 4. $\left[H^{\prime}\left(n_{1}\right)\right](k)$ is a closed subset of $(H \times X)(k)$.

Proof. Using $\operatorname{pr}_{12}, \mathscr{O}_{H \times X \times X} / \mathcal{F}_{n_{1} \Delta}^{\prime}$ is a coherent $\mathscr{O}_{H \times X}$-module. But $\mathscr{O}_{\mathscr{S}}=$ $\mathscr{O}_{H \times X \times X} / \mathscr{I}$ is a quotient of it, hence it is also a coherent $\mathscr{O}_{H \times X}$-module. This implies that $H \times X \rightarrow \mathbb{N}:(Z ; P) \rightarrow \operatorname{deg}(\mathscr{S}(Z ; P))$ is upper semicontinuous on $H \times X$ (see, e.g., [6, pp. 1-10]). Since $\left(\begin{array}{c}n_{1}+d-1 \\ d\end{array}\right)$ is the maximal possible value, we obtain our claim.

Corollary. Since $X$ is a projective variety, the projection $H \times X \stackrel{\mathrm{pr}_{1}}{\rightarrow} H$ is closed. So $\operatorname{pr}_{1}\left(\left[H^{\prime}\left(n_{1}\right)\right](k)\right) \subset H(k)$ is closed. Clearly $H(\mathbf{n}) \subset \operatorname{pr}_{1}\left(\left[H^{\prime}\left(n_{1}\right)\right](k)\right)$.

We consider now the following situation:

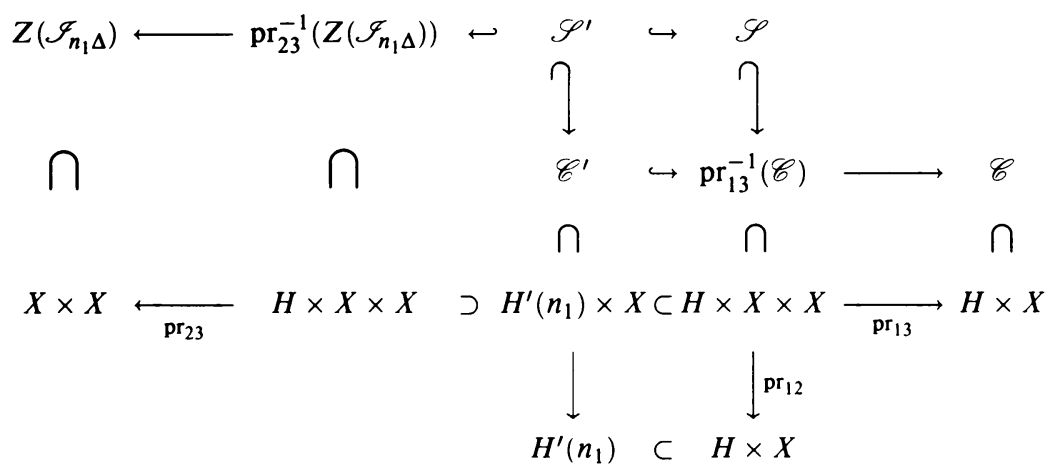

(here $Z$ means closed subscheme defined by the mentioned sheaf of ideals and $\mathscr{C}^{\prime}$ (resp. $\mathscr{S}^{\prime}$ ) are restrictions-scheme theoretical-of $\operatorname{pr}_{13}^{-1}(\mathscr{C})$ (resp. $\mathscr{S}$ ) to $\left.H^{\prime}\left(n_{1}\right) \times X\right)$. Let $\mathscr{I}_{\left(n_{1}+1\right) \Delta}^{\prime}=\mathscr{I}_{\left(n_{1}+1\right) \Delta} \cdot \mathscr{O}_{H^{\prime}\left(n_{1}\right) \times X}$ using $\mathrm{pr}_{23}$. Let $\mathscr{I}_{1}=$ $\mathscr{I}_{\left(n_{1}+1\right) \Delta}^{\prime}+\mathscr{S}_{\mathscr{C}}$ in $\mathscr{O}_{H^{\prime}\left(n_{1}\right) \times X}$ and let $\mathscr{S}_{1} \subset H^{\prime}\left(n_{1}\right) \times X$ be the associated closed subscheme. The projection on $H^{\prime}\left(n_{1}\right)$ gives a morphism $q_{1}: \mathscr{S}_{1} \rightarrow H^{\prime}\left(n_{1}\right)$.

Lemma $2^{\prime}$. For $(Z ; P) \in\left[H^{\prime}\left(n_{1}\right)\right](k)-q_{1}^{-1}(Z ; P)$ scheme-theoretical and considered as a closed subscheme of $X$-has a sheaf of ideals $\mathscr{I}_{1}(Z ; P)<\mathscr{O}_{X}$ given by

$$
\begin{aligned}
& \left(\mathscr{I}_{1}(Z ; P)\right)_{Q}=\mathscr{O}_{X ; Q} \quad \text { for } Q \in X(k) \backslash\{P\}, \\
& \left(\mathscr{I}_{1}(Z ; P)\right)_{P}=\mathscr{I}_{Z ; P}+\mathfrak{M}_{X ; P}^{n_{1}+1} .
\end{aligned}
$$

The proof is the same as the proof of Lemma 2 .

Since $\left(\mathscr{I}_{1}(Z ; P)\right)_{P} \subset(\mathscr{I}(Z ; P))_{P}$, one has $\operatorname{deg}\left(\mathscr{S}_{1}(Z ; P)\right) \geq\left(\begin{array}{c}n_{1}+d-1 \\ d\end{array}\right)$ for each $(Z ; P) \in\left[H^{\prime}\left(n_{1}\right)\right](k)$. Moreover, being a quotient of $\mathscr{O}_{H^{\prime}\left(n_{1}\right) \times X} / \mathscr{I}_{\left(n_{1}+1\right) \Delta}^{\prime}$, $\mathscr{O}_{\mathscr{S}_{1}}$ is coherent as an $\mathscr{O}_{H^{\prime}\left(n_{1}\right)}$-module. This implies that

$$
H^{\prime \prime}\left(n_{1}\right)=\left\{(Z ; P) \in H^{\prime}\left(n_{1}\right): \operatorname{deg}\left(\mathscr{S}_{1}(Z ; P)\right)>\left(\begin{array}{c}
n_{1}+d-1 \\
d
\end{array}\right)\right\} \subset H^{\prime}\left(n_{1}\right)
$$

is closed. So $H^{\prime \prime}\left(n_{1}\right) \subset H \times X$ is closed and $\operatorname{pr}_{1}\left(H^{\prime \prime}\left(n_{1}\right)\right) \subset H$ is closed. For $Z \in\left[\operatorname{pr}_{1}\left(H^{\prime \prime}\left(n_{1}\right)\right)\right](k)$, there exists $P \in X(k)$ with $l\left(\mathscr{O}_{Z ; P}\right)>\left(\begin{array}{c}n_{1}+d-1 \\ d\end{array}\right)$, so 
$Z \notin H(\mathbf{n})$. This implies

$$
H(\mathbf{n}) \subset\left[\operatorname{pr}_{1}\left(H^{\prime}\left(n_{1}\right)\right)\right](k) \backslash\left[\operatorname{pr}_{1}\left(H^{\prime \prime}\left(n_{1}\right)\right)\right](k) .
$$

We write $H\left(n_{1}\right)=H^{\prime}\left(n_{1}\right) \backslash H^{\prime \prime}\left(n_{1}\right)$.

Lemma 5. For $(Z ; P) \in\left[H\left(n_{1}\right)\right](k)$, one has $\mathscr{I}_{Z ; P}=\mathfrak{M}_{X ; P}^{n_{1}}$

Proof. Because of Lemma 3, one has $\mathscr{I}_{Z ; P} \subset \mathfrak{M}_{X ; P}^{n_{1}}$. This implies $\mathscr{I}_{Z ; P}+$ $\mathfrak{M}_{X ; P}^{n_{1}+1} \subset \mathfrak{M}_{X ; P}^{n_{1}}$. Moreover,

$$
l\left(\mathscr{O}_{X ; P} / \mathscr{I}_{Z ; P}+\mathfrak{M}_{X ; P}^{n_{1}+1}\right)=l\left(\mathscr{O}_{X ; P} / \mathfrak{M}_{X ; P}^{n_{1}}\right),
$$

hence $\mathscr{I}_{Z ; P}+\mathfrak{M}_{X ; P}^{n_{1}+1}=\mathfrak{M}_{X ; P}^{n_{1}}$. This implies

$$
\mathfrak{M}_{X ; P}^{n_{1}} / \mathscr{I}_{Z ; P}=\mathfrak{M}_{X ; P} \cdot\left(\mathfrak{M}_{X ; P}^{n_{1}} / \mathscr{I}_{Z ; P}\right)
$$

So Nakayama's Lemma implies $\mathscr{I}_{Z ; P}=\mathfrak{M}_{X ; P}^{n_{1}}$.

Let $S=\operatorname{Supp}\left(\left.\mathscr{S}_{1}\right|_{H\left(n_{1}\right) \times X}\right)$ and let $\mathscr{C}\left(n_{1}\right)$ be the scheme-theoretical restriction of $\mathscr{C}^{\prime}$ to $H\left(n_{1}\right) \times X$. Clearly $S \subset \mathscr{C}\left(n_{1}\right)$ is a union of irreducible components of $\mathscr{C}\left(n_{1}\right)$. Let $\tilde{\mathscr{C}}\left(n_{1}\right)$ be the scheme-theoretical closure of $\mathscr{C}\left(n_{1}\right) \cap$ $\left(H\left(n_{1}\right) \times X \backslash S\right)$ in $H\left(n_{1}\right) \times X$. Since $\tilde{\mathscr{C}}\left(n_{1}\right)$ is a closed subscheme of $\mathscr{C}\left(n_{1}\right)$ and $\mathscr{\mathscr { O }}_{\mathscr{E}\left(n_{1}\right)}$ is a coherent $\mathscr{O}_{H\left(n_{1}\right)}$-module $\left(\mathscr{C}\left(n_{1}\right) \rightarrow H\left(n_{1}\right)\right.$ is a projective morphism with finite fibres, so it is a finite morphism), $\mathscr{O}_{\widetilde{\mathscr{C}}\left(n_{1}\right)}$ is a coherent $\mathscr{O}_{H\left(n_{1}\right)}$-module. For a general closed point $(Z ; P)$ on some irreducible component of $H\left(n_{1}\right)$ one has

$$
\operatorname{deg}\left(\left[\widetilde{\mathscr{C}}\left(n_{1}\right)\right](Z ; P)\right)=\sum\left(l\left(\mathscr{O}_{Z ; Q}\right): Q \in X(k) \backslash\{P\}\right)=n-\left(\begin{array}{c}
n_{1}+d-1 \\
d
\end{array}\right) .
$$

So, because of semicontinuity (see again [6, pp. 1-10]), we obtain for each $(Z ; P) \in\left[H\left(n_{1}\right)\right](k)$ that $\operatorname{deg}\left(\left[\widetilde{\mathscr{C}}\left(n_{1}\right)\right](Z ; P)\right) \geq n-\left(\begin{array}{c}n_{1}+d-1 \\ d\end{array}\right):=n^{\prime}$.

Lemma 6. For each $(Z ; P) \in\left[H\left(n_{1}\right)\right](k)$ one has

$$
\operatorname{deg}\left(\left[\tilde{\mathscr{C}}\left(n_{1}\right)\right](Z ; P)\right)=n^{\prime} .
$$

Proof. Suppose for some $(Z ; P) \in\left[H\left(n_{1}\right)\right](k)$ that one has $\operatorname{deg}\left(\left[\tilde{\mathscr{C}}\left(n_{1}\right)\right](Z ; P)\right)$ $>n^{\prime}$. Since $\left[\tilde{\mathscr{C}}\left(n_{1}\right)\right](Z ; P) \subset\left[\mathscr{C}\left(n_{1}\right)\right](Z ; P)$ and $\sum\left(l\left(\mathscr{O}_{Z ; Q}\right): Q \in X(k) \backslash\{P\}\right)$ $=n^{\prime}$, this implies $(Z ; P ; P) \in\left[\widetilde{\mathscr{C}}\left(n_{1}\right)\right](k)$, so the closure of some irreducible component of $\mathscr{C}\left(n_{1}\right) \cap\left(H\left(n_{1}\right) \times X \backslash S\right)$ intersects $S$. Let $T$ be the local ring of some irreducible component of $H\left(n_{1}\right)$ containing $(Z ; P)$ at $(Z ; P)$. We write o for the closed point of $\operatorname{Spec}(T)$ and $\eta$ for the generic point of $\operatorname{Spec}(T)$. The closed subscheme $\mathscr{C}\left(n_{1}\right)$ gives rise to a finite morphism $q: \mathscr{Z} \rightarrow \operatorname{Spec}(T)$ with $\mathscr{Z}$ a closed subscheme of $\operatorname{Spec}(T) \times X$ and $\mathscr{Z}(0)=Z \subset X$. Moreover, $S$ gives rise to a section $\mathscr{S}$ of $q$ with $\mathscr{S}(0)=P$ and $\mathscr{S}_{\mathscr{Z}(\eta) ; \mathscr{S}(\eta)}=\mathfrak{M}_{X \times \operatorname{Spec}(k(\eta)) ; \mathscr{P}(\eta)}^{n_{1}}$. Moreover, the scheme-theoretical closure of $\mathscr{Z}(\eta) \backslash \mathscr{S}(\eta)$ in $\operatorname{Spec}(T) \times X$ contains $P=\mathscr{S}(0)$. Using a suited covering of $\operatorname{Spec}(T)$ we can assume that each point of $\mathscr{Z}(\eta) \backslash \mathscr{S}(\eta)$ is $k(\eta)$-rational. There exists $V \in \mathscr{Z}(\eta) \backslash \mathscr{S}(\eta)$, giving rise to a section $\mathscr{V}$ of $q$ (because the closure is contained in $\mathscr{Z}$ ), with $\mathscr{V}(0)=P=\mathscr{S}(0)$. Let $\mathscr{Z}^{\prime}$ be the scheme-theoretical closure of the restriction 
of $\mathscr{Z}$ to the local rings of $\mathscr{S}(\eta) \cup \mathscr{V}(\eta)$ in $\operatorname{Spec}(T) \times X$. Since $\mathscr{O}_{\mathscr{X}^{\prime}}$ is a quotient sheaf of $\mathscr{O}_{\mathscr{X}}$, we know that $\mathscr{O}_{\mathcal{X}^{\prime}}$ is coherent as a $\mathscr{O}_{\operatorname{Spec}(T)}$-module. Once more, from [6, pp. 1-10], we obtain

$$
\operatorname{deg}\left(\mathscr{Z}^{\prime}(\circ)\right) \geq \operatorname{deg}\left(\mathscr{Z}^{\prime}(\eta)\right) \geq\left(\begin{array}{c}
n_{1}+d-1 \\
d
\end{array}\right)+1 .
$$

But $\operatorname{Supp}\left(\mathscr{Z}^{\prime}(\circ)\right)=\{P\}$, so $l\left(\mathscr{O}_{\mathscr{X}^{\prime}(0) ; P}\right) \geq\left(\begin{array}{c}n_{1}+d-1 \\ d\end{array}\right)+1$. But since $\mathscr{Z}^{\prime} \subset \mathscr{Z}$ as schemes and $l\left(\mathscr{O}_{\mathscr{Z}(\circ) ; P}\right)=l\left(\mathscr{O}_{Z ; P}\right)=\left(\begin{array}{c}n_{1}+d-1 \\ d\end{array}\right)$, we obtain a contradiction.

Let $\widetilde{H}$ be an irreducible component of $H\left(n_{1}\right)$. Let $\tilde{\mathscr{C}}$ be the restriction of $\tilde{\mathscr{C}}\left(n_{1}\right)$ to $\tilde{H} \times X$. We obtained for each $x \in \widetilde{H}$ that $\operatorname{deg}(\tilde{\mathscr{C}}(x))=n^{\prime}$ (we proved it only for closed points, but then semicontinuity, i.e., again [6, pp. 1-10], implies the claim for all points). From [3, Theorem 9.9, p. 261], it follows that $\tilde{\mathscr{C}}\left(n_{1}\right) \rightarrow \widetilde{H}$ is a flat family of 0-dimensional subschemes of $X$ of degree $n^{\prime}$. This gives rise to natural morphisms $\tilde{\phi}: \widetilde{H} \rightarrow \operatorname{Hilb}^{n^{\prime}}(X)$ and those morphisms glue together to give a morphism $\phi: H\left(n_{1}\right) \rightarrow$ Hilb $^{n^{\prime}}(X)$. This map is described as follows on sets: $\phi(Z ; P)=Z \cap(X \backslash\{P\}) \subset X$. Clearly $Z \in H(\mathbf{n})$ if and only if there exists $P \in X(k)$ with $(Z ; P) \in H\left(n_{1}\right)$ and $\phi(Z ; P) \in H\left(\mathbf{n}^{\prime}\right)$ (we abbreviate $\left(n_{2} ; \ldots ; n_{t}\right)$ to $\mathbf{n}^{\prime}$ ) Using $\operatorname{pr}_{1}: H \times X \rightarrow H$ we obtain $H(\mathbf{n})=$ $\operatorname{pr}_{1}\left(\phi^{-1}\left(H\left(\mathbf{n}^{\prime}\right)\right)\right)$. This implies that $H(\mathbf{n})$ is locally closed in $H(k)$. Indeed, the induction hypothesis gives us $H\left(\mathbf{n}^{\prime}\right)=V_{1}^{\prime} \backslash V_{2}^{\prime}$ for some closed subsets $V_{1}^{\prime}$ and $V_{2}^{\prime}$ of $\operatorname{Hilb}^{n^{\prime}}(X)$. Let $V_{1}$ (resp. $\left.V_{2}\right)$ be the closure of $\phi^{-1}\left(V_{1}^{\prime}\right)$ (resp. $\phi^{-1}\left(V_{2}^{\prime}\right)$ ) in $(H \times X)(k)$. Then $\operatorname{pr}_{1}\left(V_{1}\right)$ and $\operatorname{pr}_{1}\left(V_{2}\right)$ are closed subsets of $H(k)$. Let $V=\operatorname{pr}_{1}\left(H^{\prime}\left(n_{1}\right)\right) \cap \operatorname{pr}_{1}\left(V_{1}\right)$ and let $V^{\prime}=\operatorname{pr}_{1}\left(H^{\prime \prime}\left(n_{1}\right)\right) \cup \operatorname{pr}_{1}\left(V_{2}\right)$.

Claim. $H(\mathbf{n})=V \backslash V^{\prime}$.

Proof. Let $Z \in H(\mathbf{n})$. Take $P \in X(k)$ with $\mathscr{I}_{Z ; P}=\mathfrak{M}_{X ; P}^{n_{1}}$. Then $(Z ; P) \in$ $H\left(n_{1}\right)$. Let $Z^{\prime}=Z \cap(X \backslash\{P\})$. Then $Z^{\prime} \in H\left(\mathbf{n}^{\prime}\right)$ and $Z^{\prime}=\phi(Z ; P)$. This implies $Z \in V$.

Assume $Z \in V^{\prime}$. Since $Z \notin H^{\prime \prime}\left(n_{1}\right)$, this implies $Z \in \operatorname{pr}_{1}\left(V_{2}\right)$. Hence there exists $P^{\prime} \in X(k)$ with $\left(Z ; P^{\prime}\right) \in V_{2}$. Since $V_{2} \subset H^{\prime}\left(n_{1}\right)$, we have $\mathscr{I}_{Z ; P^{\prime}} \subset$ $\mathfrak{M}_{X ; P^{\prime}}^{n_{1}}$, and since $Z \notin \operatorname{pr}_{1}\left(H^{\prime \prime}\left(n_{1}\right)\right)$, we have $\left(Z ; P^{\prime}\right) \in V_{2} \cap H\left(n_{1}\right)=\phi^{-1}\left(V_{2}^{\prime}\right)$ and $\mathscr{I}_{Z ; P^{\prime}}=\mathfrak{M}_{X ; P^{\prime}}^{n_{1}}$. But since $Z \in H(\mathbf{n})$ one necessarily has $\phi\left(Z ; P^{\prime}\right) \in$ $H\left(\mathbf{n}^{\prime}\right)$, so $\phi\left(Z ; P^{\prime}\right) \notin V_{2}^{\prime}$, a contradiction. This proves $H(\mathbf{n}) \subset V \backslash V^{\prime}$

Conversely, let $Z \in V \backslash V^{\prime}$. Since $Z \in V \subset \operatorname{pr}_{1}\left(H^{\prime}\left(n_{1}\right)\right) \cap \operatorname{pr}_{1}\left(V_{1}\right)$, there exists $P \in X(k)$ with $\mathscr{I}_{Z ; P} \subset \mathfrak{M}_{X ; P}^{n_{1}}$ and $(Z ; P) \in V_{1}$. Since $Z \notin V^{\prime \prime} \supset$ $\operatorname{pr}_{1}\left(H^{\prime \prime}\left(n_{1}\right)\right)$, this implies $\mathscr{I}_{Z ; P}=\mathfrak{M}_{X ; P}^{n_{1}}$, hence $(Z ; P) \in H\left(n_{1}\right) \cap V_{1}=$ $\phi^{-1}\left(V_{1}^{\prime}\right)$, so $\phi(Z ; P) \in V_{1}^{\prime}$. Since $Z \notin V^{\prime \prime} \supset \operatorname{pr}_{1}\left(V_{2}\right)$, we have $\phi(Z ; P) \notin V_{2}^{\prime}$, so $\phi(Z ; P) \in V_{1}^{\prime} \backslash V_{2}^{\prime}=H\left(\mathbf{n}^{\prime}\right)$. This implies $Z \in H(\mathbf{n})$.

This completes the proof of the claim and the theorem.

\section{REFERENCES}

1. M. V. Catalisano, Linear systems of plane curves through fixed "fat" points of $\mathbb{P}^{2}, \mathrm{~J}$. Algebra 142 (1991), 81-100.

2. A. Gimigliano, Our thin knowledge of fat points, The Curves Seminar at Queen's 6; Queen's Papers in Pure and Appl. Math. 83 (1989).

3. R. Hartshorne, Algebraic geometry, Graduate Texts in Math., vol. 52, Springer-Verlag, Berlin and New York, 1977. 
4. D. Mumford, Lectures on curves on an algebraic surface, Ann. of Math. Stud., no 59, Princeton Univ. Press, Princeton, NJ, 1966.

5. G. Paxia, On flat families of fat points, Proc. Amer. Math. Soc. 112 (1991), 19-23.

6. E. Sernesi, Topics on families of projective schemes, Queen's Papers in Pure and Appl. Math. 73 (1986).

Katholieke Industriële Hogeschool der Kempen, Campus H. I. Kempen, KleinhoefsTRAAT 4, B2440 GeEL, BELgium

E-mail address: FGABA11 at CC1.KuLeuven.ac.be 